Commun. Fac. Sci. Univ. Ank. Ser. A1 Math. Stat.

Volume 68, Number 2, Pages 1506-1527(2019)

DOI: $10.31801 /$ cfsuasmas. 542988

ISSN 1303-5991 E-ISSN 2618-6470

http://communications.science.ankara.edu.tr/index.php?series=A1

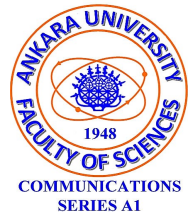

\title{
THE TOPP-LEONE GENERALIZED ODD LOG-LOGISTIC FAMILY OF DISTRIBUTIONS: PROPERTIES, CHARACTERIZATIONS AND APPLICATIONS
}

\author{
M. KORKMAZ, H. M. YOUSOF, M. ALIZADEH, AND G.G. HAMEDANI
}

\begin{abstract}
A new family of distributions called the Topp-Leone generalized odd log-logistic-G family is introduced and studied. We provide some mathematical properties of the new family including ordinary and incomplete moments, generating function and order statistics. We assess the performance of the maximum likelihood estimators in terms of biases and mean squared errors by means of two simulation studies. Finally, the usefulness of the family is illustrated by means of two real data sets. The new model provides consistently better fits than other competitive models for these data sets.
\end{abstract}

\section{INTRODUCTION}

Various continuous univariate models have been extensively used for modeling data in many areas. So, several families of distributions have been constructed by extending common classes of continuous distributions. These generalized distributions give high flexibility by adding one or more parameters to the baseline model. The main goal of this article is to propose a new class of distributions from the Topp-Leone model and the generalized odd log logistic model that can have increasing and upside down bathtub hazard rate to be used for modeling lifetime data. Recently, several properties of the Topp-Leone distribution have been investigated by several authors. The cumulative distribution function (cdf) of the Topp-Leone distribution is given by

$$
F_{T L}(x ; \alpha)=\{x(2-x)\}^{\alpha}=\left\{1-[1-x]^{2}\right\}^{\alpha},
$$

where $0<x<1$ and $\alpha>0$ is a shape parameter. Recently, several properties of the Topp-Leone distribution have been investigated by several authors such as

Received by the editors: October 05, 2017; Accepted: November 11, 2018.

2010 Mathematics Subject Classification. 60E05, 62E10, 62F10.

Key words and phrases. Topp-Leone distribution, odd log-logistic family, maximum likelihood, characterizations, generating function, order statistic, simulation.

(C)2019 Ankara University Communications Faculty of Sciences University of Ankara-Series A1 Mathematics and Statistics 
Nadarajah and Kotz (2003), Ghitany et al. (2005), Zhou et al. (2006), Kotz and Seier (2007), Zghoul (2011), Gen012) and Gen013).

On the other hand, generalized odd log-logistic (GOLL-G) family defined by Cordeiro et al. (2017) has the following cdf

$$
H_{G O L L-G}(x ; \beta, \theta, \boldsymbol{\psi})=G(x ; \boldsymbol{\psi})^{\beta \theta}\left\{G(x ; \boldsymbol{\psi})^{\beta \theta}+\left[1-G(x ; \boldsymbol{\psi})^{\theta}\right]^{\beta}\right\}^{-1},
$$

where $\beta, \theta>0$ are shape parameters and $G(x ; \boldsymbol{\psi})$ is the baseline cdf with parameter vector $\boldsymbol{\psi}$. For $\theta=1$, the odd log-logistic-G (OLL-G) family, defined by Gleaton and Lynch (2006), is obtained. The GOLL-G family is more flexible than OLL-G family.

Many odd log-logistic-G families can be cited such as the Kumaraswamy odd loglogistic family of distributions by Alizadeh et al. (2015), the Zografos-Balakrishnan odd log-logistic family of distributions by Cordeiro et al. (2016a), the beta odd loglogistic generalized family of distributions by Cordeiro et al. (2016b), the generalized odd log-logistic family of distributions by Cordeiro et al. (2017), the another generalized odd log-logistic family of distributions by Haghbin et al. (2017), the Topp-Leone odd log-logistic G (TLOLL-G) family of distributions by Brito et al. (2017) and the exponential Lindley odd log-logistic G family by Korkmaz et al. (2018).

This paper is organized as follows. In Section 2, we defined the new family. In Section 3, we provide two special TLGOLL-G distributions. In Section 4, several of its mathematical properties are derived. Section 5 provides some useful characterization results. The maximum likelihood inference of the model parameters is performed in Section 6 as well as two simulation studies are presented for maximum likelihood estimations of the parameters in Section 7. Applications to two real data sets illustrating the performance of the methodology have been proposed in Section 8. The paper is concluded in Section 9.

\section{THE NEW FAMILY}

In this Section, we define a new flexible family of distributions with various types of hazard rate and density flexibility. A method of generating families of distributions is to combine with $F(H)$ structure which have the cdf as the value of the cdf of the distribution $F$ whose range is the unit interval $H$. With this idea, by using equations (1) and (2), we can define the cdf of the new family by

$$
F(x ; \alpha, \beta, \theta, \boldsymbol{\psi})=\left(1-\left\{\frac{\left[1-G(x ; \boldsymbol{\psi})^{\theta}\right]^{\beta}}{G(x ; \boldsymbol{\psi})^{\beta \theta}+\left[1-G(x ; \boldsymbol{\psi})^{\theta}\right]^{\beta}}\right\}^{2}, \quad x \in \mathbb{R},\right.
$$

where $\alpha, \beta, \theta>0$ are the additional shape parameters which ensure the flexibility of the model. The pdf corresponding to Equation (3) is given by 


$$
\begin{gathered}
f(x ; \alpha, \beta, \theta, \boldsymbol{\psi})=\frac{2 \alpha \beta \theta g(x ; \boldsymbol{\psi}) G(x ; \boldsymbol{\psi})^{\beta \theta-1}\left[1-G(x ; \boldsymbol{\psi})^{\theta}\right]^{2 \beta-1}}{\left\{G(x ; \boldsymbol{\psi})^{\beta \theta}+\left[1-G(x ; \boldsymbol{\psi})^{\theta}\right]^{\beta}\right\}^{3}} \\
\times\left(1-\left\{1-\frac{G(x ; \boldsymbol{\psi})^{\beta \theta}}{G(x ; \boldsymbol{\psi})^{\beta \theta}+\left[1-G(x ; \boldsymbol{\psi})^{\theta}\right]^{\beta}}\right\}^{2}, x \in \mathbb{R} .\right.
\end{gathered}
$$

For $\theta=1$, the TLGOLL-G family is reduced to TLOLL-G family. Hereafter, the random variable $X$ is denoted by $X \sim$ TLGOLL-G $(\alpha, \beta, \theta, \psi)$. Further, we can omit the dependence on the vector $\boldsymbol{\psi}$ of the parameters and write simply $G(x ; \boldsymbol{\psi})=G(x)$ and $g(x ; \boldsymbol{\psi})=g(x)$. The hazard rate function (hrf) of $X$ is given by

$$
h(x)=\frac{\frac{2 \alpha \beta \theta g(x) G(x)^{\beta \theta-1}\left[1-G(x)^{\theta}\right]^{2 \beta-1}}{\left\{G(x)^{\beta \theta}+\left[1-G(x)^{\theta}\right]^{\beta}\right\}^{3}}\left(1-\left\{1-\frac{G(x)}{G(x)^{\beta \theta}+\left[1-G(x)^{\theta}\right]^{\beta}}\right\}^{2}\right)^{\alpha-1}}{1-\left(1-\left\{1-\frac{G(x)^{\beta \theta}}{G(x)^{\beta \theta}+\left[1-G(x)^{\theta}\right]^{\beta}}\right\}^{2}\right)^{\alpha}}, x \in \mathbb{R} .
$$

The quantile function of $X$ is given by

$$
x_{u}=G^{-1}\left(\left\{\frac{\left[\left(1-u^{1 / \beta}\right)^{-0.5}-1\right]^{1 / \alpha}}{\left[\left(1-u^{1 / \beta}\right)^{-0.5}-1\right]^{1 / \alpha}+1}\right\}^{1 / \theta}\right)
$$

where $G^{-1}(\cdot)$ is the inverse of the baseline cdf. Hence, If $U$ is a uniform random variable on $(0,1)$, then $X_{U}$ has TLGOLL-G distribution.

\section{Two Special Members of the Family}

Here, we obtain two special sub-models of the new family. These special models extend some well-known distributions given in the literature.

3.1. The TLGOLL-normal (TLGOLL-N) distribution. To extend the normal distribution, we consider TLGOLL-N distribution as first example by taking $G(x ; \mu, \sigma)=\Phi\left(\frac{x-\mu}{\sigma}\right)$ and $g(x ; \mu, \sigma)=\phi\left(\frac{x-\mu}{\sigma}\right)$ to be the cdf and pdf in (3), where $x, \mu \in \mathbb{R}, \sigma>0, \phi(\cdot)$ and $\Phi(\cdot)$ are the pdf and cdf of the standard normal distribution, respectively. The cdf of the TLGOLL-N distribution is given by

$$
F(x ; \alpha, \beta, \theta, \mu, \sigma)=\left(1-\left\{\frac{\left[1-\Phi\left(\frac{x-\mu}{\sigma}\right)^{\theta}\right]^{\beta}}{\Phi\left(\frac{x-\mu}{\sigma}\right)^{\beta \theta}+\left[1-\Phi\left(\frac{x-\mu}{\sigma}\right)^{\theta}\right]^{\beta}}\right\}^{2}, x \in \mathbb{R} .\right.
$$

Some possible plots of the TLGOLL-N density and hrf for selected parameter values are displayed in Figure 1. This figure shows that the pdf shapes of the TLGOLL-N can be skewed, bi-modal and uni-modal shaped. Also, its hrf shapes are increasing or firstly increasing shaped then bathtub shaped. 

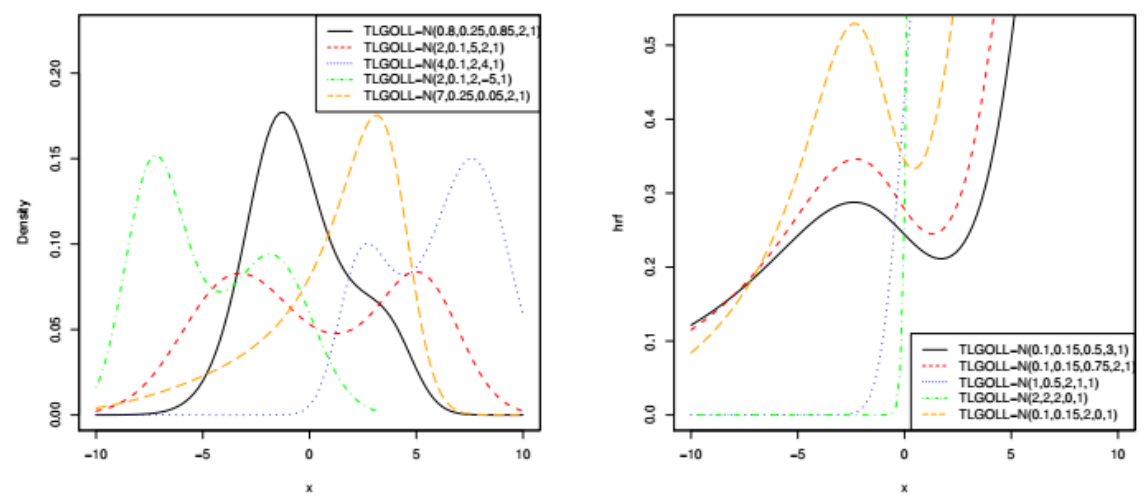

Figure 1. The pdf and hrf of the TLGOLL-N distribution for selected parameter values

3.2. The TLGOLL-Weibull (TLGOLL-W) distribution. As a second submodel, we consider the Weibull distribution with $\operatorname{cdf} G(x ; \lambda, \gamma)=1-\exp \left[-(\lambda x)^{\gamma}\right]$ for $x>0$ and $\lambda, \gamma>0$. The cdf of the TLGOLL-W distribution is given by

$F(x ; \alpha, \beta, \theta, \lambda, \gamma)=\left(1-\left\{\frac{\left[1-\left(1-e^{-(\lambda x)^{\gamma}}\right)^{\theta}\right]^{\beta}}{\left(1-e^{-(\lambda x)^{\gamma}}\right)^{\beta \theta}+\left[1-\left(1-e^{-(\lambda x)^{\gamma}}\right)^{\theta}\right]^{\beta}}\right\}^{2}, x>0\right.$.
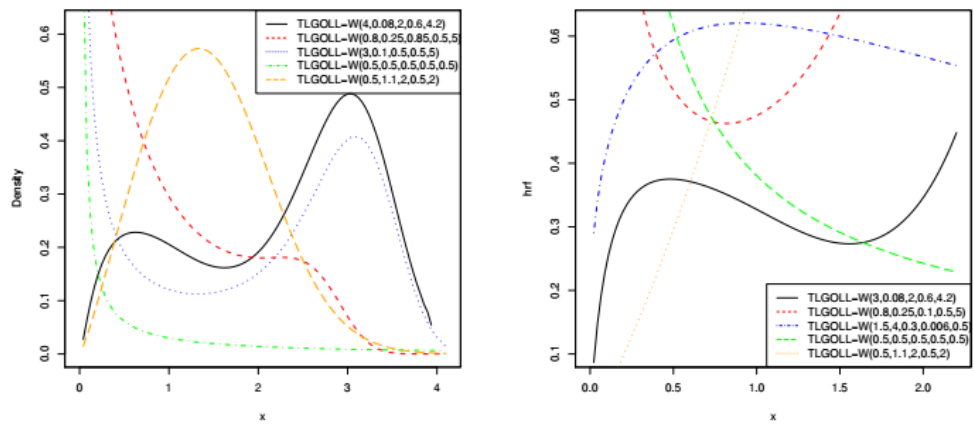

Figure 2. The pdf and hrf of the TLGOLL-W distribution for selected parameter values

Some possible plots of the TLGOLL-W density and hrf for selected parameter values are displayed in Figure 2. Figure 2 shows that the pdf shapes can be 
bi-modal, uni-modal, decreasing, firstly U shaped then decreasing shaped. Nevertheless, its hrf shapes can be bathtube shaped, uni-modal shaped, firstly increasing shaped then bathtub shaped, decreasing and increasing shaped.

From these results, we can say that the TLGOLL-G family can generate very flexible distributions for data modeling.

\section{Some mathematical Properties}

4.1. Useful expansions. Using the generalized binomial expansion the cdf in (3) can be written as

$$
\begin{aligned}
F(x) & =\sum_{i=0}^{\infty}(-1)^{i}\left(\begin{array}{c}
\alpha \\
i
\end{array}\right)\left[1-\frac{G(x)^{\beta \theta}}{G(x)^{\beta \theta}+\left[1-G(x)^{\beta}\right]^{\theta}}\right]^{2 i} \\
& =\sum_{i=0}^{\infty} \sum_{j=0}^{2 i}(-1)^{i+j}\left(\begin{array}{c}
\alpha \\
i
\end{array}\right)\left(\begin{array}{c}
2 i \\
j
\end{array}\right) \underbrace{\frac{\overparen{G(x)^{\beta \theta j}}}{\left\{G(x)^{\beta \theta}+\left[1-G(x)^{\beta}\right]^{\theta}\right\}^{j}}}_{B} .
\end{aligned}
$$

Expanding $A$ and $B$ as

$$
\frac{A}{B}=\frac{\sum_{k=0}^{\infty} a_{k} G(x)^{k}}{\sum_{k=0}^{\infty} b_{k} G(x)^{k}}=\sum_{k=0}^{\infty} c_{k} G(x)^{k}
$$

where

$$
a_{k}=\sum_{l=k}^{\infty}(-1)^{l+k}\left(\begin{array}{c}
\beta \theta j \\
l
\end{array}\right)\left(\begin{array}{l}
l \\
k
\end{array}\right)
$$

and $b_{k}=h_{k}(\beta, \theta, 2 i)$, the quantity $h_{k}(\beta, \theta, 2 i)$ defined in Appendix $\mathrm{A}$ and $c_{0}=$ $a_{0}\left(b_{0}\right)^{-1}$ and for $k \geq 1$ we have

$$
c_{k}=\left(b_{0}\right)^{-1}\left[a_{k}-\left(b_{0}\right)^{-1} \sum_{r=1}^{k} b_{r} c_{k-r}\right] .
$$

Finally, the cdf of the TLGOLL-G family can be expressed as

$$
F(x)=\sum_{k=0}^{\infty} d_{k} G(x)^{k}=\sum_{k=0}^{\infty} d_{k} \Pi_{k}(x)
$$

where

$$
d_{k}=\sum_{i=0}^{\infty} \sum_{j=0}^{2 i}(-1)^{i+j}\left(\begin{array}{c}
\alpha \\
i
\end{array}\right)\left(\begin{array}{c}
2 i \\
j
\end{array}\right) c_{k}(\beta, \theta, i, j)
$$


and $\Pi_{\gamma}(x)=G(x)^{\gamma}$ is the exp-G cdf with power parameter $\gamma>0$. By differentiating Equation (6), we obtain the density function of $\mathrm{X}$ as

$$
f(x)=\sum_{k=0}^{\infty} d_{k+1} \pi_{k+1}(x)
$$

where $\pi_{\gamma}(x)=\gamma g(x) G(x)^{\gamma-1}$ is the exp-G pdf with power parameter $\gamma>0$. Equation (7) reveals that the TLGOLL-G density function is a linear combination of exp-G densities. Based on this equation, we can obtain some statistical quantities for the new family from the corresponding ones of the exp-G model.

4.2. Moments, incomplete moments and generating function. The $\mathrm{r}^{\text {th }}$ ordinary moment of $X$ is given by $E\left(X^{r}\right)=\mu_{r}^{\prime}=\int_{-\infty}^{\infty} x^{r} f(x) d x$. Then we obtain

$$
\mu_{r}^{\prime}=\sum_{k=0}^{\infty} d_{k+1} E\left(Y_{k+1}^{r}\right)
$$

Henceforth, $Y_{\gamma}$ denotes the exp-G random variable with power parameter $\gamma$. For $\gamma>0$, we have $E\left(Y_{\gamma}^{r}\right)=\gamma \int_{-\infty}^{\infty} x^{r} g(x) G(x)^{\gamma-1} d x$, which can be computed numerically in terms of the baseline quantile function (qf) $Q_{G}(u)=G^{-1}(u)$ as $E\left(Y_{\gamma}^{n}\right)=\gamma \int_{0}^{1} Q_{G}(u)^{n} u^{\gamma-1} d u$. Setting $r=1$ in (8), we have the mean of $X$. The last integration can be computed numerically for most parent distributions. The skewness and kurtosis measures can be calculated from the ordinary moments using well-known relationships. The $\mathrm{n}^{\text {th }}$ central moment of $X$, say $M_{n}$, is $M_{n}=E(X-\mu)^{n}=\sum_{h=0}^{n}(-1)^{h}\left(\begin{array}{l}n \\ h\end{array}\right)\left(\mu_{1}^{\prime}\right)^{n} \mu_{n-h}^{\prime}$. The cumulants $\left(\kappa_{n}\right)$ of $X$ follow recursively from $\kappa_{n}=\mu_{n}^{\prime}-\sum_{r=0}^{n-1}\left(\begin{array}{c}n-1 \\ r-1\end{array}\right) \kappa_{r} \mu_{n-r}^{\prime}$, where $\kappa_{1}=\mu_{1}^{\prime}$, $\kappa_{2}=\mu_{2}^{\prime}-\mu_{1}^{\prime 2}, \kappa_{3}=\mu_{3}^{\prime}-3 \mu_{2}^{\prime} \mu_{1}^{\prime}+\mu_{1}^{\prime 3}$, etc. The skewness and kurtosis measures also can be calculated from the ordinary moments using well-known relationships. The main applications of the first incomplete moment refer to the mean deviations and the Bonferroni and Lorenz curves. These curves are very useful in demography, economics, reliability, insurance and medicine. The $\mathrm{r}^{t h}$ incomplete moment, say $I_{r}(t)$, of $X$, can be expressed from (7) as

$$
I_{r}(t)=\int_{-\infty}^{t} x^{r} f(x) d x=\sum_{k=0}^{\infty} d_{k+1} \int_{-\infty}^{t} x^{r} \pi_{k+1}(x) d x .
$$

The first incomplete moment $I_{1}(t)$ can be obtained from (9) with $r=1$. A general equation for $I_{1}(t)$ can be derived from $(9)$ as $I_{1}(t)=\sum_{k=0}^{\infty} d_{k+1} J_{k+1}(x)$, where $J_{\gamma}(x)=\int_{-\infty}^{t} x \pi_{\gamma}(x) d x$ is the first incomplete moment of the exp-G model. The moment generating function (mgf) $M_{X}(t)=E\left(e^{t X}\right)$ of $X$ can be derived from equation (7) as $M_{X}(t)=\sum_{k=0}^{\infty} d_{k+1} M_{k+1}(t)$, where $M_{\gamma}(t)$ is the $\operatorname{mgf}$ of $Y_{\gamma}$. Hence, 
$M_{X}(t)$ can be determined from the exp-G generating function. For the TLGOLL$\mathrm{W}$ model, we have

$$
\mu_{r}^{\prime}=\Gamma(1+r / \gamma) \sum_{k, w=0}^{\infty} v_{w, k}^{(r, k+1)}, \forall r>-\gamma
$$

and

$$
I_{r}(t)=\Gamma\left(1+r / \gamma,(\lambda / t)^{\gamma}\right) \sum_{k, w=0}^{\infty} v_{w, k}^{(r, k+1)}, \forall r>-\gamma
$$

where

$$
v_{w, k}^{(r, k+1)}=d_{k+1}(k+1)(-1)^{w} \lambda^{-r}(w+1)^{-(r+\gamma) / \gamma}\left(\begin{array}{c}
k \\
w
\end{array}\right) .
$$

We obtain skewness and kurtosis values for TLGOLL-N and TLGOLL-W distributions in Figure 3 and Table 1. It is well-known that the normal distribution has zero skewness and three kurtosis values. So, it is more effective to model on symmetrical data for inference. Hence, its modeling ability is bounded. From Figure 3 , we see that TLGOLL-N distribution can be left skewed, right skewed and symmetrical as well as having different kurtosis values from ordinary normal distribution. Table 1 shows that very different skewness and kurtosis values have been obtained for the same $\lambda$ and $\gamma$ values. Consequently, we can say that these new models can be more useful for various data sets than their ordinary models.
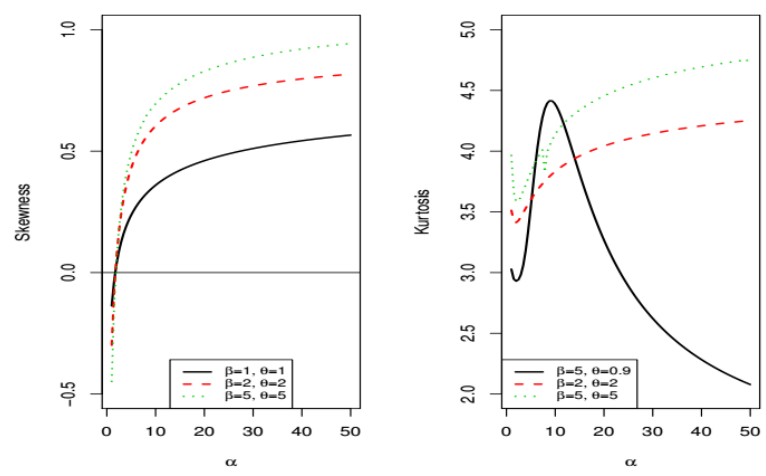

FIGURE 3. Skewness and kurtosis plots of TLGOLL-N distribution for $\mu=0$ and $\sigma=1$

4.3. Moments of residual and reversed residual life. The $n^{\text {th }}$ moment of the residual life say, $a_{n}(t)=E\left[(X-t)^{n} \mid X>t\right], n=1,2, \ldots$, uniquely determines $F(x)$ and is given by

$$
a_{n}(t)=\frac{1}{1-F(t)} \int_{t}^{\infty}(x-t)^{n} d F(x) .
$$


TABLE 1. Skewness and kurtosis values for special TLGOLL-W and $\mathrm{W}$ distributions

\begin{tabular}{lcc|cc}
\hline \hline Parameters & \multicolumn{2}{c}{ TLGOLL $-W(\alpha, \beta, \theta, \lambda, \gamma)$} & \multicolumn{2}{c}{$W(\lambda, \gamma)$} \\
\hline$\alpha, \beta, \theta, \lambda, \gamma$ & Skewness & Kurtosis & Skewness & Kurtosis \\
\hline $0.5,1,0.5,1,0.5$ & 15.3611 & 467.4255 & 6.6187 & 87.7200 \\
\hline $2,1,1,0.5,1$ & 1.6099 & 7.0800 & 2 & 9 \\
\hline $2,2,5,5,5$ & 0.0265 & 3.4042 & -0.2542 & 2.8803 \\
\hline $2,2,2,0.5,0.5$ & 1.9323 & 11.1769 & 6.6187 & 87.7200 \\
\hline $5,5,5,10,5$ & 173.4906 & 30100 & -0.2541 & 2.8803 \\
\hline $1.2,15,5,2,2$ & -0.3628 & 3.9412 & 0.6311 & 3.2451 \\
\hline \hline
\end{tabular}

Therefore

$$
a_{n}(t)=\sum_{k=0}^{\infty} \sum_{r=0}^{n} \frac{d_{k+1}(1-t)^{n}}{1-F(t)} \int_{t}^{\infty} x^{r} \pi_{k+1}(x) d x .
$$

The $n^{\text {th }}$ moment of the reversed residual life say, $A_{n}(t)=E\left[(t-X)^{n} \mid X \leq t\right]$ for $t>0$ and $n=1,2, \ldots$ uniquely determines $F(x)$. We obtain $A_{n}(t)=\frac{1}{F(t)} \int_{0}^{t}(t-$ $x)^{n} d F(x)$. Then, the $n^{t h}$ moment of the reversed residual life of $X$ becomes

$$
A_{n}(t)=\sum_{k=0}^{\infty} \sum_{r=0}^{n} \frac{d_{k+1}}{F(t)}(-1)^{r}\left(\begin{array}{l}
n \\
r
\end{array}\right) t^{n-r} \int_{0}^{t} x^{r} \pi_{k+1}(x) d x .
$$

The mean residual life (MRL) function or the life expectation at age $t$ defined by $z_{1}(t)=E[(X-t) \mid X>t]$, which represents the expected additional life length for a unit which is alive at age $t$. The MRL of $X$ can be obtained by when $n=1$ in $A_{n}(t)$ equation. For the TLGOLL-W model we have

$$
a_{n}(t)=\frac{\Gamma\left(1+n / \gamma,(\lambda / t)^{\gamma}\right)}{[1-F(t)]} \sum_{k, w=0}^{\infty} \sum_{r=0}^{n} v_{w, k, r}^{(n, k+1)}, \forall n>-\gamma,
$$

and

$$
A_{n}(t)=\frac{\Gamma\left(1+n / \gamma,(\lambda / t)^{\gamma}\right)}{F(t)} \sum_{k, w=0}^{\infty} \sum_{r=0}^{n} \vartheta_{w, k, r}^{(n, k+1)}, \forall n>-\gamma,
$$

where

$$
v_{w, k, r}^{(n, k+1)}=d_{k+1}(k+1)(-1)^{w}(1-t)^{n} \lambda^{-n}(w+1)^{-(n+\gamma) / \gamma}\left(\begin{array}{c}
k \\
w
\end{array}\right),
$$

and

$$
\vartheta_{w, k, r}^{(n, k+1)}=d_{k+1}(k+1)(-1)^{w+r} t^{n-r} \lambda^{-n}(w+1)^{-(n+\gamma) / \gamma}\left(\begin{array}{l}
n \\
r
\end{array}\right)\left(\begin{array}{l}
k \\
w
\end{array}\right) .
$$


4.4. Order statistics. Suppose $X_{1}, \ldots, X_{n}$ is a random sample from any TLGOLLG model and let $X_{i: n}$ denote the $\mathrm{i}^{\text {th }}$ order statistic. The pdf of $X_{i: n}$ can be expressed as

$$
f_{i: n}(x)=\frac{f(x)}{B(i, n-i+1)} \sum_{j=0}^{n-i}(-1)^{j}\left(\begin{array}{c}
n-i \\
j
\end{array}\right) F(x)^{j+i-1} .
$$

We can write the density function of $X_{i: n}$ as

$$
f_{i: n}(x)=\sum_{l, k=0}^{\infty} d_{l, k} h_{l+k+1}(x),
$$

where

$$
d_{l, k}=\frac{n !(i-1) !(l+1)}{(l+k+1)} d_{l+1} \sum_{j=0}^{n-i} \frac{(-1)^{j}}{(n-i-j) ! j !} \zeta_{j+i-1, k}
$$

and the quantities $\zeta_{j+i-1, k}$ can be determined with $\zeta_{j+i-1,0}=d_{0}^{j+i-1}$ and recursively for $k \geq 1, \zeta_{j+i-1, k}=\left(k d_{0}\right)^{-1} \sum_{m=1}^{k}[m(j+i)-k] d_{m} \zeta_{j+i-1, k-m}$. Equation (10) is the main result of this section. It reveals that the pdf of the TLGOLL-G order statistics is a linear combination of exp-G density functions. So, several mathematical quantities of the TLGOLL-G order statistics such as ordinary, incomplete and factorial moments, mean deviations and several others can be determined from those quantities of the exp-G distribution. For the TLGOLL-W model we have

$$
E\left(X_{i: n}^{q}\right)=\Gamma(1+q / \gamma) \sum_{l, k, w=0}^{\infty} v_{l, k, w}^{(q, l+k+1)}, \forall q>-\gamma,
$$

where

$$
v_{l, k, w}^{(q, l+k+1)}=d_{l, k}(l+k+1)(-1)^{w} \lambda^{-q}(w+1)^{-(q+\gamma) / \gamma}\left(\begin{array}{c}
l+k \\
w
\end{array}\right) .
$$

\section{Characterization}

This section deals with certain characterizations of TLGOLL-G distribution. These characterizations are in terms of: $(i)$ two truncated moments and $(i i)$ conditional expectations of functions of the random variable. One of the advantages of characterization $(i)$ is that the cdf is not required to have a closed form. Due to the nature of our cdf, we believe our characterizations may be the only possible ones. We present our characterizations $(i)$ and $(i i)$ in two subsections.

5.1. Characterizations based on two truncated moments. In this subsection we present characterizations of TLGOLL-G distribution in terms of a simple relationship between two truncated moments. This characterization result employs a theorem due to Glel (1987), see Theorem 1 of Appendix B. Note that the result holds also when the interval $H$ is not closed. Moreover, as mentioned above, it could also be applied when the cdf $F$ does not have a closed form. As shown in Glel (1990), this characterization is stable in the sense of weak convergence. 
Proposition 5.1. Let $X: \Omega \rightarrow \mathbb{R}$ be a continuous random variable and let $q_{1}(x)=\frac{G(x)^{\theta(1-\beta)}\left(G(x)^{\beta \theta}+\left[1-G(x)^{\theta}\right]^{\beta}\right)^{3}}{\left[1-\left(\frac{\left[1-G(x)^{\theta}\right]^{\beta}}{G(x)^{\beta \theta}+\left[1-G(x)^{\theta}\right]^{\beta}}\right)^{2}\right]^{\alpha-1}}$ and $q_{2}(x)=q_{1}(x)\left[1-G(x)^{\theta}\right]$ for $x \in \mathbb{R}$. The random variable $X$ has pdf (4) if and only if the function $\eta$ defined in Theorem 1 has the form

$$
\eta(x)=\frac{2 \beta}{2 \beta+1}\left[1-G(x)^{\theta}\right], \quad x \in \mathbb{R} .
$$

Proof. Let $X$ be a random variable with pdf (4), then

$$
(1-F(x)) E\left[q_{1}(X) \mid X \geq x\right]=\alpha\left[1-G(x)^{\theta}\right]^{2 \beta}, \quad x \in \mathbb{R},
$$

and

$$
(1-F(x)) E\left[q_{2}(X) \mid X \geq x\right]=\frac{2 \alpha \beta}{2 \beta+}\left[1-G(x)^{\theta}\right]^{2 \beta+1}, \quad x \in \mathbb{R},
$$

and finally

$$
\eta(x) q_{1}(x)-q_{2}(x)=-\frac{1}{2 \beta+1} q_{1}(x)\left[1-G(x)^{\theta}\right]<0 \quad \text { for } \quad x \in \mathbb{R} .
$$

Conversely, if $\eta$ is given as above, then

$$
s^{\prime}(x)=\frac{\eta^{\prime}(x) q_{1}(x)}{\eta(x) q_{1}(x)-q_{2}(x)}=\frac{2 \beta \theta g(x) G(x)^{\theta-1}}{\left[1-G(x)^{\theta}\right]} \quad x \in \mathbb{R},
$$

and hence

$$
s(x)=\log \left\{\left[1-G(x)^{\theta}\right]^{-2 \beta}\right\}, \quad x \in \mathbb{R} .
$$

Now, in view of Theorem 1, $X$ has pdf (4).

Corollary 5.1. Let $X: \Omega \rightarrow \mathbb{R}$ be a continuous random variable and let $q_{1}(x)$ be as in Proposition 5.1. The pdf of $X$ is (4) if and only if there exist functions $q_{2}$ and $\eta$ defined in Theorem 1 satisfying the differential equation

$$
\frac{\eta^{\prime}(x) q_{1}(x)}{\eta(x) q_{1}(x)-q_{2}(x)}=\frac{2 \beta \theta g(x) G(x)^{\theta-1}}{\left[1-G(x)^{\theta}\right]} \quad x \in \mathbb{R} .
$$

The general solution of the differential equation in Corollary 5.1 is

$$
\eta(x)=\left[1-G(x)^{\theta}\right]^{-1}\left[-\int 2 \beta \theta g(x) G(x)^{\theta-1}\left(q_{1}(x)\right)^{-1} q_{2}(x)+D\right],
$$


where $D$ is a constant. Note that a set of functions satisfying the above differential equation is given in Proposition 5.1 with $D=0$. However, it should be also noted that there are other triplets $\left(q_{1}, q_{2}, \eta\right)$ satisfying the conditions of Theorem 1.

5.2. Characterization based on the conditional expectation of certain functions of the random variable. In this subsection we employ a single function $\psi$ of $X$ and characterize the distribution of $X$ in terms of the truncated moment of $\psi(X)$. The following proposition has already appeared in Hamedani's previous work (2013), so we will just state it here as a proposition, which can be used to characterize TLGOLL-G distribution for $\alpha=1$.

Proposition 5.2. Let $X: \Omega \rightarrow(d, e)$ be a continuous random variable with cdf $F$. Let $\psi(x)$ be a differentiable function on $(d, e)$ with $\lim _{x \rightarrow e^{-}} \psi(x)=1$. Then for $\delta \neq 1$,

$$
E[\psi(X) \mid X \geq x]=\delta \psi(x), \quad x \in(d, e)
$$

if and only if

$$
\psi(x)=(1-F(x))^{\frac{1}{\delta}-1}, \quad x \in(d, e) .
$$

Remark 5.2. For $\alpha=1,(d, e)=\mathbb{R}, \psi(x)=\left(\frac{\left[1-G(x)^{\theta}\right]^{\beta}}{G(x)^{\beta \theta}+\left[1-G(x)^{\theta}\right]^{\beta}}\right)$ and $\delta=\frac{2}{3}$, Proposition 5.2 provides a characterization of TLGOLL-G distribution.

\section{Maximum Likelihood Estimations (MLEs) of the parameters}

Several approaches for parameter estimation were proposed in the literature but the maximum likelihood method is the most commonly employed. The MLEs enjoy desirable properties and can be used for constructing confidence intervals and also for test statistics. The normal approximation for these estimators in large samples can be easily handled either analytically or numerically. Here, we consider the estimation of the unknown parameters of the new family from complete samples only by maximum likelihood Method. Let $x_{1}, \ldots, x_{n}$ be a random sample from TLGOLL-G model with a $(q+3) \times 1$ parameter vector $\boldsymbol{\Xi}=(\alpha, \beta, \theta, \boldsymbol{\psi})^{\top}$, where $\boldsymbol{\psi}$ is a $q \times 1$ baseline parameter vector. The log-likelihood function for $\boldsymbol{\Xi}$ is

$$
\begin{aligned}
\ell(\boldsymbol{\Xi})= & \log 2+\log \alpha+\log \beta+\log \theta+\sum_{i=0}^{n} \log g\left(x_{i} ; \boldsymbol{\psi}\right) \\
& +(\beta \theta-1) \sum_{i=0}^{n} \log G\left(x_{i} ; \boldsymbol{\psi}\right)+(2 \beta-1) \sum_{i=0}^{n} \log \left[1-G\left(x_{i} ; \boldsymbol{\psi}\right)^{\theta}\right] \\
& -3 \sum_{i=0}^{n} \log \left\{G\left(x_{i} ; \boldsymbol{\psi}\right)^{\beta \theta}+\left[1-G\left(x_{i} ; \boldsymbol{\psi}\right)^{\theta}\right]^{\beta}\right\}
\end{aligned}
$$




$$
+(\alpha-1) \sum_{i=0}^{n} \log \left[1-\left(1-\frac{G\left(x_{i} ; \boldsymbol{\psi}\right)^{\beta \theta}}{G\left(x_{i} ; \boldsymbol{\psi}\right)^{\beta \theta}+\left[1-G\left(x_{i} ; \boldsymbol{\psi}\right)^{\theta}\right]^{\beta}}\right)^{2}\right] .
$$

Setting the nonlinear system of equations $U(\alpha)=U(\beta)=U(\theta)=U\left(\boldsymbol{\psi}_{r}\right)=$ 0 (for $r=1=\ldots, q$ ) and solving them simultaneously yields the MLEs $\widehat{\Xi}=$ $\left(\widehat{\alpha}, \widehat{\beta}, \widehat{\theta}, \widehat{\boldsymbol{\psi}}^{\top}\right)^{\top}$. To solve these equations, it is more convenient to use nonlinear optimization methods such as the quasi-Newton algorithm to numerically maximize $\ell(\Xi)$.

The likelihood ratio (LR) statistic can be used for comparing the TLGOLL-G model with TLOLL-G model, which is equivalently to test $H_{0}: \theta=1$. For this situaiton, the LR statistic is computed with $w=2[\ell(\hat{\alpha}, \hat{\beta}, \hat{\theta}, \hat{\boldsymbol{\psi}})-\ell(\tilde{\alpha}, \tilde{\beta}, 1, \tilde{\boldsymbol{\psi}})]$, where $(\hat{\alpha}, \hat{\beta}, \hat{\theta}, \hat{\boldsymbol{\psi}})$ are the unrestricted MLEs and $(\tilde{\alpha}, \tilde{\beta}, 1, \tilde{\boldsymbol{\psi}})$ are the restricted estimates under $H_{0}$. The statistic $w$ is asymptotically (as $\left.n \rightarrow \infty\right)$ distributed as $\chi_{v}^{2}$, where $v$ is difference of two parameter vectors of nested models. For example, $v=1$ for above hypothesis test.

\section{Simulation studies}

In this Section, we perform two simulation studies by using the TLGOLL-W and TLGOLL-N distributions to see the performance of the MLEs corresponding to these distribution. The random numbers generation is obtained by the inverse of their cdfs. All results related to MLEs were obtained using optim-CG routine in the $\mathrm{R}$ programme.

7.1. Simulation study 1 . In the first simulation study, we obtain the graphical results. We generate $N=1000$ samples of size $n=20,25,30, \ldots, 1000$ from TLGOLL-W distribution with parameters values $\alpha=4, \beta=2, \theta=8, \lambda=0.1$ and $\gamma=2$. We calculate the empirical mean, standard deviation (sd), bias and mean square errors (MSE) of the MLEs. The bias and MSE are calculated by (for $h=\alpha, \beta, \theta, \lambda, \gamma)$

$$
\widehat{\operatorname{Bias}_{h}}=\frac{1}{N} \sum_{i=1}^{N}\left(\hat{h}_{i}-h\right)
$$

and

$$
\widehat{M S E_{h}}=\frac{1}{N} \sum_{i=1}^{N}\left(\hat{h}_{i}-h\right)^{2}
$$

respectively. We give results of this simulation study in Figure 4 From Figure 4. we observe that when the sample size increases, the empirical means approach the true parameter value whereas all biases, sds and MSEs approach to 0 in all cases. 

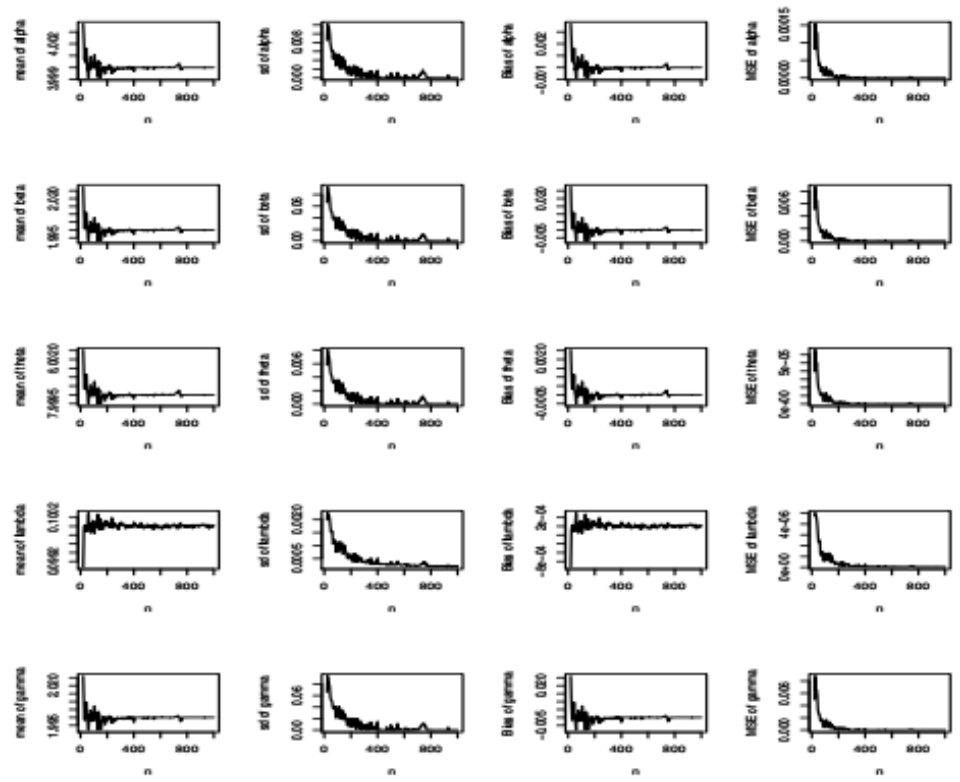

FIGURE 4. Simulation results of the special TLGOLL-W distribution

TABLE 2. The empirical means and standard deviations (in parentheses) for the special TLGOLL-N distributions.

\begin{tabular}{|c|c|c|c|c|c|c|c|c|c|c|c|c|c|c|c|}
\hline & \multicolumn{5}{|c|}{$n=20$} & \multicolumn{5}{|c|}{$n=100$} & \multicolumn{5}{|c|}{$n=150$} \\
\hline $\bar{\alpha}, \beta, \theta, \mu, \sigma$ & $\widehat{\hat{\alpha}}$ & $\tilde{\beta}$ & $\tilde{\theta}$ & $\hat{\mu}$ & $\hat{\sigma}$ & $\widehat{\hat{\alpha}}$ & $\bar{\beta}$ & $\tilde{\theta}$ & $\hat{\mu}$ & $\hat{\sigma}$ & $\hat{a}$ & $\hat{\beta}$ & $\tilde{\theta}$ & $\hat{\mu}$ & $\hat{\sigma}$ \\
\hline $2,2,2,0,2$ & 2.1969 & 1.9187 & 2.3502 & 0.2405 & 1.4841 & 1.9650 & 1.9280 & 2.0761 & 0.0521 & 1.8634 & 2.0007 & 1.9580 & 2.0693 & 0.0510 & 1.9757 \\
\hline 0.52 .050 .051 & (1.5.253) & $\frac{(.0 .007)}{2.0227}$ & $\frac{(3.3857)}{0.4829}$ & (1.7.727x) & 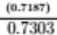 & (1.0.177) & 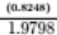 & 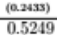 & (0.3965) & (a.s50) & 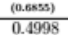 & (195x) & (0.0.514) & (0.2.2509) & $\frac{(0.2077)}{0.9219}$ \\
\hline & & & & & & & & (a.0sas) & & & (0.2455) & & & & \\
\hline $0.5,1,-1,2$ & 4.9567 & 0.4634 & 1.4203 & 1.1996 & 1.8201 & 4.9724 & 0.5118 & 1.1327 & 1.0630 & 2.0521 & 4.9853 & & 1.1211 & & \\
\hline & & & & & & & & & & & & & & & \\
\hline $1,2,0.5,-1,0.5$ & 1.1053 & 1.9170 & 0.5130 & 1.0812 & 0.4153 & 1.0116 & 1.9450 & 0.5121 & -1.0265 & 0.4758 & 1.0114 & 1.9579 & 0.5028 & -1.0242 & 0.4821 \\
\hline & 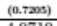 & (a.2502) & 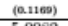 & (0.1935) & 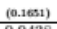 & 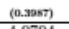 & 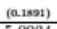 & (1.0.0587) & & & $(0.3502)$ & $\frac{(1.1590)}{0.150)}$ & (2) & $\frac{(0.0763)}{(0.03)}$ & 190 \\
\hline $5,5,5,0,1$ & 4.9710 & 5.0222 & 5.0082 & 0.0646 & 0.9438 & 4.9794 & 5.0034 & 4.9992 & 0.0045 & 0.9978 & & 5.0066 & 5.0032 & 0.0017 & 0.9949 \\
\hline $5,4,3,2,1$ & 5.1484 & 4.0032 & 3.0259 & $\overline{1222}$ & 0.9733 & & 022 & 0043 & 2.00 & 0.9911 & & 0.0010 & 3.0011 & 2.0059 & 0.9985 \\
\hline & (1.005n) & 13:so) & (a.1900) & & & $(0.250)$ & & & & & (0.20.201) & & (20030) & & \\
\hline-10.3 & 3.0030 & 0.9290 & 2.0036 & 0.4932 & 0.0902 & 3.0016 & 0.9949 & 2.0027 & 0.4998 & 0.0995 & 2.9980 & 1.0034 & 1.9984 & .500 & .1006 \\
\hline & (1.0.000x) & (1.18772) & (OAsos) & (20.015s) & 1.02210) & (a.assa) & 1250) & (cosis) & (bass) & .01010) & noan) & of(12) & (2.032) & (atose) & oass \\
\hline
\end{tabular}

7.2. Simulation study 2 . In the second simulation study, we generate 1,000 samples of sizes 20, 100 and 150 from selected TLGOLL-N distributions. For this simulation study, we obtain the empirical means and sd's of the MLEs. The results of this simulation study are reported in Table 2. Table 2 shows that when the sample size increases, the empirical means approach true parameter value whereas the sds decrease in all the cases as expected. 
As a results, we can say that the MLE method works very well to estimate the parameters of the TLGOLL-G distribution.

\section{EMPIRICAL APPLICATIONS}

In this section, we illustrate the flexibility of the TLGOLL-N and TLGOLL$\mathrm{W}$ models via two data sets. We also compare these models with others models which are well-known in the literature. To determine the optimum model, we also compute the estimated log-likelihood values $\hat{\ell}$, Akaike Information Criteria (AIC), corrected Akaike information criterion (CAIC), Bayesian information criterion (BIC), Hannan-Quinn information criterion (HQIC), Cramer von Mises $\left(W^{*}\right)$ and Anderson-Darling $\left(A^{*}\right)$ goodness of-fit statistics for all models. We note that the AIC, CAIC, BIC and HQIC are given by $A I C=-2 \widehat{\ell}+2 p, C A I C=$ $-2 \widehat{\ell}+2 p n(n-k-1)^{-1}, B I C=-2 \widehat{\ell}+p \log n$ and $H Q I C=-2 \widehat{\ell}+p \log (\log n)$, where $p$ is the number of the estimated model parameters and $n$ is the sample size. The $W^{*}$ and $A^{*}$ statistics can be calculated as

$$
W^{*}=\sum_{i=1}^{n}\left(\hat{F}\left(x_{(i)}\right)-\frac{i-0.5}{n}\right)^{2}+\frac{1}{12 n}
$$

and

$$
A^{*}=-\sum_{i=1}^{n} \frac{2 i-1}{n}\left[\ln \hat{F}\left(x_{(i)}\right)+\ln \hat{\bar{F}}\left(x_{(n+1-i)}\right)\right]-n .
$$

The statistics $W^{*}$ and $A^{*}$ are described in detail in Chen and Balakrishnan (1995) and Evans et al. (2008). In general, it can be chosen as the best model which has the smaller values of the AIC, CAIC, BIC, HQIC, $W^{*}$ and $A^{*}$ statistics and the larger values of $\hat{\ell}$. MLEs computations are performed by the maxLik routine and the statistics $W^{*}$ and $A^{*}$ are obtained by the goftest routine in the $\mathrm{R}$ programme. The details are given below.

8.1. Otis IQ Scores of non-white males data set. The first real data set is the Otis IQ Scores of 52 non-white males hired by a large insurance company in 1971. This data set has been analyzed by Roberts (1988), Gupta and Gupta (2004), Sharafi and Behboodian (2008) and Jamalizadeh et al. (2011). The data are: 91, 102, 100, 117, 122, 115, 97, 109, 108, 104, 108, 118, 103,123, 123, 103, 106, 102, $118,100,103,107,108,107,97,95,119,102,108,103,102,112,99,116,114,102$, $111,104,122,103,111,101,91,99,121,97,109,106,102,104,107,95$. For this data set, we compare the TLGOLL-N model with the N model, TLOLL-N model, beta normal (B-N) model (Eugene et al., 2002), Marshall-Olkin normal (MO-N) model (Garcia et al., 2010), Kumaraswamy normal (Kw-N) model (Cordeiro and Castro, 2011), McDonald normal (Mc-N) model (Alexander et al., 2012) and odd log-logistic normal (OLL-N) model (Braga et al., 2016). 
8.2. Voltage data. The second data set, studied by Meeker and Escobar (1998, p. 383), gives the times of failure and running times for a sample of devices from a field-tracking study of a larger system. The data are: 275, 13, 147, 23, 181, 30, $65,10,300,173,106,300,300,212,300,300,300,2,261,293,88,247,28,143$, $300,23,300,80,245,266$. This data also analyzed by Cordeiro et al. (2010) and Alexander et al. (2012).

The total time test (TTT) plot due to Aarset (1987) is an important graphical approach to verify whether the data can be applied to a specific distribution or not. According to Aarset (1987), the empirical version of the TTT plot is given by plotting $T(r / n)=\left[\sum_{i=1}^{r} y_{i: n}+(n-r) y_{r: n}\right] / \sum_{i=1}^{n} y_{i: n}$ against $r / n$, where $r=$ $1, \ldots, n$ and $y_{i: n}(i=1, \ldots, n)$ are the order statistics of the sample. Aarset (1987) showed that the hazard function is constant if the TTT plot is graphically presented as a straight diagonal, the hazard function is increasing (or decreasing) if the TTT plot is concave (or convex). The hazard function is U-shaped (bathtub) if the TTT plot is firstly convex and then concave, if not, the hazard function is unimodal. The TTT plots for Voltage data set is presented in Figure 5. This plot indicates that the empirical hazard rate functions of the data set is U-shaped (bathtub).

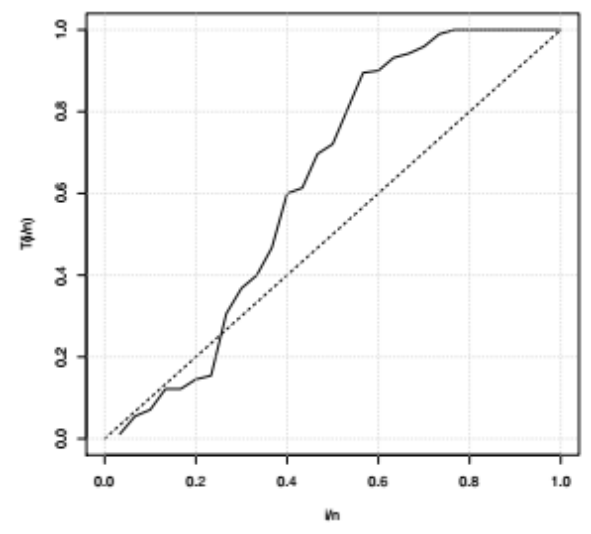

Figure 5. TTT plot for Voltage data

For this data set, we compare the TLGOLL-W model with the TLOLL-W model, W model, exponentiated Weibull (E-W) model (Mudholkar and Srivastava, 1993), Marshall-Olkin Weibull (MO-W) model (Marshall and Olkin, 1997), beta Weibull (B-W) model (Famoye et al., 2005), Kumaraswamy Weibull (Kw-W) model (Cordeiro et al., 2010) and McDonald Weibull (Mc-W) model (Alexander et al., 2012).

Table 3 lists the MLEs, their standard erros of the parameters and $\hat{\ell}$ values from the fitted models and Table 4 shows AIC, CAIC, BIC, HQIC, $W^{*}$ and $A^{*}$ statistics for both data sets. The TGLOLL-N and TLGOLL-W models could be chosen as 
the best model among the fitted models since these models have the lowest values of the AIC, CAIC, HQIC, $W^{*}$ and $A^{*}$ statistics and have the biggest $\hat{\ell}$ values.

TABLE 3. MLEs, standard erros of the estimates (in parentheses) and $\hat{\ell}$ for the applications models

\begin{tabular}{|c|c|c|c|c|c|c|c|}
\hline Data Set & Model & $\widehat{\alpha}$ & $\widehat{\beta}$ & $\widehat{\theta}$ & $\widehat{\mu}$ & $\widehat{\sigma}$ & $-\hat{\ell}$ \\
\hline \multirow[t]{17}{*}{$\mathrm{I}$} & TLGOLL-N & 0.8261 & 0.0480 & 0.3747 & 115.4490 & 0.9407 & 177.5493 \\
\hline & & $(0.2414)$ & $(0.0112)$ & $(0.1201)$ & $(0.0025)$ & $(0.0026)$ & \\
\hline & TLOLL-N & 0.7770 & 0.0829 & - & 113.3936 & 1.7777 & 179.0879 \\
\hline & & $(0.1857)$ & $(0.0152)$ & & $(0.2795)$ & $(0.0384)$ & \\
\hline & $\mathrm{Mc}-\mathrm{N}$ & 0.0160 & 0.0573 & 2.7977 & 111.0082 & 1.4883 & 179.2420 \\
\hline & & $(0.0023)$ & $(0.0132)$ & $(0.0315)$ & $(0.0001)$ & $(0.0001)$ & \\
\hline & $\mathrm{B}-\mathrm{N}$ & 2.4062 & 0.2218 & - & 93.3939 & 5.7048 & 182.1709 \\
\hline & & $(0.6739)$ & 0.0337 & & $(0.0250)$ & $(0.0439)$ & \\
\hline & $\mathrm{Kw}-\mathrm{N}$ & 6.4121 & 0.4031 & - & 89.6488 & 8.5717 & 182.2576 \\
\hline & & $(2.3057)$ & 0.1434 & & $(0.8662)$ & $(1.5830)$ & \\
\hline & MO-N & 0.2507 & - & - & 112.8747 & 8.3928 & 182.3138 \\
\hline & & $(0.2446)$ & & & $(4.7639)$ & $(0.9630)$ & \\
\hline & OLL-N & 0.5051 & - & - & 107.4663 & 4.8560 & 183.0290 \\
\hline & & $(0.2968)$ & & & $(1.5178)$ & $(2.0013)$ & \\
\hline & $\mathrm{N}$ & - & - & - & 106.6537 & 8.2288 & 183.3872 \\
\hline & & & & & $(1.1637)$ & $(0.8150)$ & \\
\hline & & & & & $\widehat{\lambda}$ & $\widehat{\gamma}$ & \\
\hline \multirow[t]{16}{*}{ II } & TLGOLL-W & 2.9534 & 0.0385 & 1.4733 & 0.0054 & 7.3178 & 162.3933 \\
\hline & & $(0.5680)$ & $(0.0056)$ & $(0.0078)$ & $(0.00002)$ & $(0.0077)$ & \\
\hline & TLOLL-W & 3.7055 & 0.0575 & - & 0.0055 & 6.3932 & 169.5266 \\
\hline & & $(0.7083)$ & $(0.0082)$ & & $(0.0001)$ & $(0.0004)$ & \\
\hline & Mc-W & 0.0359 & 0.0390 & 1.7500 & 0.0052 & 7.9997 & 168.6354 \\
\hline & & $(0.0080)$ & $(0.0088)$ & $(0.1384)$ & $(0.0001)$ & $(0.0020)$ & \\
\hline & B-W & 0.0751 & 0.0592 & - & 0.0051 & 7.9097 & 169.7702 \\
\hline & & $(0.0175)$ & 0.0128 & & $(0.0001)$ & $(0.0007)$ & \\
\hline & $\mathrm{Kw}-\mathrm{W}$ & 0.0488 & 0.2095 & - & 0.0043 & 7.6793 & 172.0609 \\
\hline & & $(0.0230)$ & 0.0814 & & $(0.0003)$ & $(0.0005)$ & \\
\hline & MO-W & 5.5194 & - & - & 0.0116 & 0.9564 & 182.7515 \\
\hline & & $(6.9393)$ & & & $(0.0084)$ & $(0.3064)$ & \\
\hline & E-W & 0.1168 & - & - & 0.0030 & 7.0202 & 176.9930 \\
\hline & & $(0.0221)$ & & & $(0.0002)$ & $(0.0012)$ & \\
\hline & $\mathrm{W}$ & - & - & - & 0.0053 & 1.2650 & 184.3138 \\
\hline & & & & & $(0.0007)$ & $(0.2042)$ & \\
\hline
\end{tabular}


TABLE 4. Information criteria results, $A^{*}$ and $W^{*}$ statistics for the applications models

\begin{tabular}{c|ccccccc}
\hline \hline Data Set & Model & $A I C$ & $C A I C$ & $B I C$ & $H Q I C$ & $A^{*}$ & $W^{*}$ \\
\hline \multirow{6}{*}{ I } & TLGOLL-N & 365.0986 & 366.4030 & 374.8548 & 368.8389 & 0.2593 & 0.0377 \\
& TLOLL-N & 366.1757 & 367.0268 & 373.9807 & 369.1679 & 0.3700 & 0.0584 \\
& Mc-N & 368.4839 & 369.7883 & 378.2401 & 372.2242 & 0.3422 & 0.0520 \\
& B-N & 372.3418 & 373.1928 & 380.1467 & 375.3340 & 0.4427 & 0.0672 \\
& Kw-N & 372.5152 & 373.3663 & 380.3202 & 375.5074 & 0.4249 & 0.0625 \\
& MO-N & 370.6275 & 371.1275 & 376.4813 & 372.8717 & 0.5009 & 0.0722 \\
& OLL-N & 372.0581 & 372.5581 & 377.9118 & 374.3023 & 1.0994 & 0.2336 \\
& N & 370.7743 & 371.0192 & 374.6768 & 372.2704 & 0.8137 & 0.1390 \\
\hline II & TLGLL-W & 334.7865 & 337.2865 & 341.7925 & 337.0278 & 0.7142 & 0.0851 \\
& TLOLL-W & 347.0531 & 348.6531 & 352.6579 & 348.8461 & 1.0526 & 0.1560 \\
& Mc-W & 347.2707 & 349.7707 & 354.2767 & 349.5120 & 0.9262 & 0.1196 \\
& B-W & 347.5404 & 349.1404 & 353.1452 & 349.3334 & 0.8173 & 0.1320 \\
& Kw-W & 352.1217 & 353.7217 & 357.7265 & 353.9148 & 1.1869 & 0.1682 \\
& MO-W & 371.5030 & 372.4361 & $375.7,66$ & 372.8478 & 1.9065 & 0.2575 \\
& E-W & 359.9859 & 360.9090 & 364.1895 & 361.3307 & 2.3032 & 0.3851 \\
& W & 372.6277 & 373.0721 & 375.4301 & 373.5242 & 2.1106 & 0.3316 \\
\hline \hline
\end{tabular}

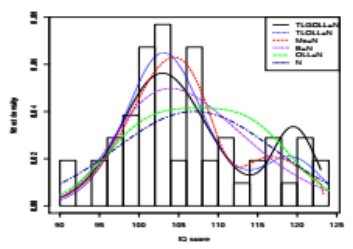

(A) Fitted pdfs for data set I

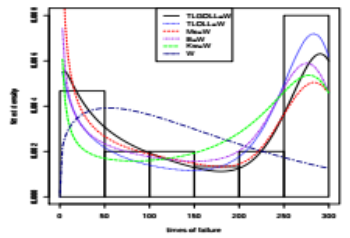

(c) Fitted pdfs for data set II

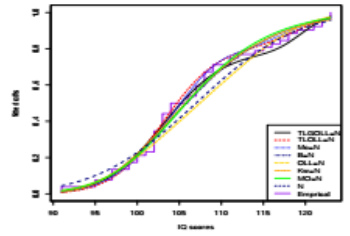

(B) Fitted cdfs for data set I

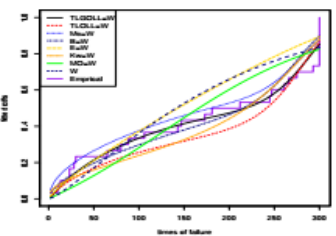

(D) Fitted cdfs for data set II

Figure 6 . The fitted pdfs and cdfs for the data sets

The plots of the fitted densities and fitted cdfs of all models are displayed in Figure 6. These plots also reveal that the TGLOLL-N and TGLOLL-W models provide the good fit to these data compared to the other models. The TLGOLL-N model fits the data set as bi-modal shaped whereas ordinary $\mathrm{N}$ model fits the data set as symmetrical bell-shaped. At the same time, The TLGOLL-W model fits the data set as firstly U-shaped then decreasing shaped whereas ordinary $\mathrm{W}$ model fits the data set as uni-modal shaped. Hence, we observe that fittings of the TLGOLL$\mathrm{N}$ and TLGOLL-W are better than the fittings of the ordinary $\mathrm{N}$ and $\mathrm{W}$ models and successfully capture the shape of the data. 


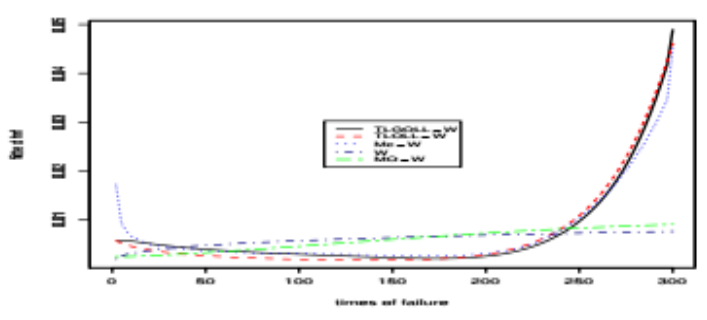

Figure 7. Fitted hrf plots

Further, we sketch the fitted hrf plots of TLGOLL-W,TLOLL-W, Mc-W, MO-W and $\mathrm{W}$ models in Figure 7. From this Figure, we see that $\mathrm{W}$ and MO-W model have fitted as increasing hrf shaped whereas TLGOLL-W model has fitted bathtub hrf shaped. This result of TLGOLL-W model deals with Figure 5

A comparison of the proposed distributions with some of their sub-models using LR statistics is performed in Table 5 . Table 5 shows that TLGOLL-G models provide a better representation of the data than the their sub-model based on the LR test at the $10 \%$ significance level. We reject the null hypotheses of two LR tests in favor of the TLGOLL-G distributions. The rejection is extremely highly significant for the voltage data as well as for the IQ data. Hence, we can say that the additional parameter is effective on sub-models.

TABLE 5. LR statistics for both data sets

\begin{tabular}{|c|c|c|c|}
\hline Model & Нyр & $w$ & p-va \\
\hline TLGOLL-N vs TLOLL-N & $H_{0}: \theta=1, H_{1}: H_{0}$ false & 3.0772 & 0.0794 \\
\hline TLGOLL-W vs TLOLL-W & $H_{0}: \theta=1, H_{1}: H_{0}$ false & 14.2666 & 0.0001 \\
\hline
\end{tabular}

\section{Conclusions}

A new family of distributions called the Topp-Leone Generalized Odd Log-logistic G family is introduced and studied. We provided some mathematical properties of the new family including ordinary and incomplete moments, generating function and order statistics. Some new useful characterization results based on two truncated moments as well as on the conditional expectation of certain functions of the random variable are provided. We assessed the performance of the maximum likelihood estimators in terms of the biases and mean squared errors by means of two simulation studies. Finally, the usefulness of the family is illustrated by means of two real data sets. The new proposed models provide consistently better fits than other competitive models on data sets. 


\section{Appendix A.}

\section{Four useful power series}

We present four power series required for the algebraic developments in Section 3. First, for $a>0$ real non-integer, we have the binomial expansion

$$
(1-u)^{a}=\sum_{j=0}^{\infty}(-1)^{j}\left(\begin{array}{l}
a \\
j
\end{array}\right) u^{j}
$$

where the binomial coefficient is defined for any real. Second after using twice generalized binomial expansion and changing the summation over $j, k$, for any $0<$ $u<1$, one can write

$$
u^{\alpha}=\sum_{k=0}^{\infty} s_{k} u^{k}
$$

where

$$
s_{k}=s_{k}(\alpha)=\sum_{i=k}^{\infty}(-1)^{i+k}\left(\begin{array}{l}
\alpha \\
i
\end{array}\right)\left(\begin{array}{l}
i \\
k
\end{array}\right) .
$$

Third, we can expand $z^{\lambda}$ in Taylor series to obtain

$$
z^{\lambda}=\sum_{k=0}^{\infty}(\lambda)_{k}(z-1)^{k}(k !)^{-1}=\sum_{i=0}^{\infty} f_{i} z^{i}
$$

where

$$
f_{i}=f_{i}(\lambda)=\sum_{k=i}^{\infty}(-1)^{k-i}(k !)^{-1}\left(\begin{array}{c}
k \\
i
\end{array}\right)(\lambda)_{k}
$$

and $(\lambda)_{k}=\lambda(\lambda-1) \ldots(\lambda-k+1)$ denotes the falling factorial. Fourth, we obtain an expansion for $\left[G(x)^{\beta \theta}+\left[1-G(x)^{\theta}\right]^{\beta}\right]^{c}$. We can write from equation (A1)

$$
\left[G(x)^{\beta \theta}+\left[1-G(x)^{\theta}\right]^{\beta}\right]=\sum_{j=0}^{\infty} t_{j} G(x)^{j},
$$

where

$$
t_{j}(\alpha, \beta)=s_{j}(\beta \theta)+\sum_{p=0}^{\infty}(-1)^{p}\left(\begin{array}{l}
\beta \\
p
\end{array}\right) s_{j}(\theta p) \text { for } j \geq 0,
$$

and $f_{j}(\alpha)$ is defined by (A2). Then, using (A3), we have

$$
\left[G(x)^{\beta \theta}+\left[1-G(x)^{\theta}\right]^{\beta}\right]^{c}=\sum_{i=0}^{\infty} f_{i}\left(\sum_{j=0}^{\infty} t_{j} G(x)^{j}\right)^{i},
$$

where $f_{i}=f_{i}(c)$. Finally, using again equations (A2) and (A3), we obtain

$$
\left[G(x)^{\beta \theta}+\left[1-G(x)^{\theta}\right]^{\beta}\right]^{c}=\sum_{j=0}^{\infty} h_{j}(\beta, \theta, c) G(x)^{j},
$$


where $h_{j}(\beta, \theta, c)=\sum_{i=0}^{\infty} f_{i} m_{i, j}$ and (for $\left.i \geq 0\right) m_{i, j}=\left(j t_{0}\right)^{-1} \sum_{m=1}^{j}[m(j+1)-$ $j] t_{m} m_{i, j-m}$ (for $j \geq 1$ ) and $m_{i, 0}=t_{0}^{i}$.

\section{Appendix B.}

Theorem 1. Let $(\Omega, \mathcal{F}, \mathbf{P})$ be a given probability space and let $H=[a, b]$ be an interval for some $d<b(a=-\infty, b=\infty$ might as well be allowed $)$. Let $X: \Omega \rightarrow$ $H$ be a continuous random variable with the distribution function $F$ and let $q_{1}$ and $q_{2}$ be two real functions defined on $H$ such that

$$
\mathbf{E}\left[q_{2}(X) \mid X \geq x\right]=\mathbf{E}\left[q_{1}(X) \mid X \geq x\right] \eta(x), \quad x \in H,
$$

is defined with some real function $\eta$. Assume that $q_{1}, q_{2} \in C^{1}(H), \eta \in C^{2}(H)$ and $F$ is twice continuously differentiable and strictly monotone function on the set $H$. Finally, assume that the equation $\eta q_{1}=q_{2}$ has no real solution in the interior of $H$. Then $F$ is uniquely determined by the functions $q_{1}, q_{2}$ and $\eta$, particularly

$$
F(x)=\int_{a}^{x} C\left|\frac{\eta^{\prime}(u)}{\eta(u) q_{1}(u)-q_{2}(u)}\right| \exp (-s(u)) d u
$$

where the function $s$ is a solution of the differential equation $s^{\prime}=\frac{\eta^{\prime} q_{1}}{\eta q_{1}-q_{2}}$ and $C$ is the normalization constant, such that $\int_{H} d F=1$.

Acknowledgment. The author would like to thank the editor and anonymous referees for carefully reading the article and for their great help in improving the article.

\section{REFERENCES}

[1] Aarset, M. V., How to identify a bathtub hazard rate. IEEE Transactions on Reliability, 36, (1987), 106-108.

[2] Alexander, C., Cordeiro, G. M., Ortega, E. M. and Sarabia, J. M., Generalized beta-generated distributions. Computational Statistics and Data Analysis, 56(6), (2012), 1880-1897.

[3] Alizadeh, M., Emadi, M., Doostparast, M., Cordeiro, G.M., Ortega, E.M.M. and Pescim, R.R., The Kumaraswamy odd log-logistic family of distributions, Hacettepe Journal of Mathematics and Statistics, 44, (2015), 1491-1512.

[4] Cordeiro, G.M., Alizadeh, M., Ortega, E.M.M. and Serrano, L.H.V., The ZografosBalakrishnan odd log-logistic family of distributions: Properties and Applications. Hacettepe Journal of Mathematics and Statistics, 45, (2016a), 1781-1803.

[5] Cordeiro, G.M., Alizadeh, M., Tahir, M. H., Mansoor, M., Bourguignon, M. and Hamedani G.G., The beta dd log-logistic generalized family of distributions, Hacettepe Journal of Mathematics and Statistics, 45, (2016b), 1175-1202.

[6] Cordeiro, G.M., Alizadeh, M., Ozel, G., Hosseini, B., Ortega, E.M.M. and Altun, E., The generalized odd log-logistic family of distributions: properties, regression models and applications, Journal of Statistical Computation and Simulation, 87, (2017), 908-932.

[7] Braga, A.S., Cordeiro, G. M., Ortega, E. M. and Nilton da Cruz, J., The odd log-logistic normal distribution: Theory and applications in analysis of experiments. Journal of Statistical Theory and Practice, 10(2), (2016), 311-335.

[8] Brito, E., Cordeiro, G. M., Yousof, H. M., Alizadeh, M.and Silva, G. O., The Topp-Leone odd log-logistic family of distributions. Journal of Statistical Computation and Simulation, 87, (2017), 3040-3058. 
[9] Chen, G. and Balakrishnan, N., A general purpose approximate goodness-of-fit test. Journal of Quality Technology, 27, (1995) 154-161.

[10] Cordeiro, G. M., Alizadeh, M., Ozel, G., Hosseini, B., Ortega, E. M. M. and Altun, E., The generalized odd log-logistic family of distributions: properties, regression models and applications. Journal of Statistical Computation and Simulation, 87, (2017), 908-932.

[11] Cordeiro, G. M. and de Castro, M., A new family of generalized distributions. Journal of statistical computation and simulation, 81, (2011), 883-898.

[12] Cordeiro, G. M., Ortega, E. M. and Nadarajah, S., The Kumaraswamy Weibull distribution with application to failure data. Journal of the Franklin Institute, 347, (2010), 1399-1429.

[13] Eugene, N., Lee, C. and Famoye, F., Beta-normal distribution and its applications. Communications in Statistics-Theory and methods, 31, (2002), 497-512.

[14] Evans, D. L., Drew, J. H. and Leemis, L. M., The distribution of the Kolmogorov-Smirnov, Cramer-von Mises, and Anderson-Darling test statistics for exponential populations with estimated parameters. Communications in Statistics-Simulation and Computation, 37, (2008) 1396-1421.

[15] Famoye, F., Lee, C. and Olumolade, O., The beta-Weibull distribution. Journal of Statistical Theory and Applications, 4, (2005), 121-136.

[16] Garcia, V. J., Gomez-Deniz, E. and Vazquez-Polo, F. J., A new skew generalization of the normal distribution: Properties and applications. Computational Statistics 83 Data Analysis, 54(8), (2010), 2021-2034.

[17] Gen., Moments of order statistics of Topp Leone distribution. Statistical Papers, 51, (2012), $1-15$.

[18] Gen., Estimation of the $\operatorname{Prob}(\mathrm{X}>\mathrm{Y})$ with Topp Leone distribution. Journal of Statistical Computation and Simulation, 83, (2013), 326-339.

[19] Ghitany, M. E., Kotz, S., Xie, M., On some reliability measures and their stochastic orderings for the Topp Leone distribution. Journal of Applied Statistics, 32, (2005), 715-722.

[20] Glel, W., A characterization theorem based on truncated moments and its application to some distribution families. Mathematical Statistics and Probability Theory (Bad Tatzmannsdorf, 1986), Vol. B, Reidel, Dordrecht, (1987), 75-84.

[21] Glel, W., Some consequences of a characterization theorem based on truncated moments. Statistics: A Journal of Theoretical and Applied Statistics, 21, (1990), 613-618.

[22] Gleaton, J. U. and Lynch, J. D., Properties of generalized log-logistic families of lifetime distributions. Journal of Probability and Statistical Science, 4, (2006), 51-64.

[23] Gupta R.C and Gupta R.D., Generalized skew normal model. Test 13, (2004), 501-524.

[24] Haghbin, H., Ozel, G., Alizadeh, M. and Hamedani, G. G., A new generalized odd log-logistic family of distributions. Communications in Statistics-Theory and Methods, 46, (2017), 98979920.

[25] Hamedani, G.G., On certain generalized gamma convolution distributions II, Technical Report No. 484, MSCS, Marquette University (2013).

[26] Jamalizadeh, A., Arabpour, A. R. and Balakrishnan, N., A generalized skew two-piece skewnormal distribution. Statistical Papers, 52, (2011), 431-446.

[27] Korkmaz, M. , Yousof, H. M. and Hamedani G. G., The exponential Lindley odd log-logistic G family: properties, characterizations and applications. Journal of Statistical Theory and Applications, 17, (2018), 554-571.

[28] Kotz, S. and Seier, E., Kurtosis of the Topp Leone distributions. Interstat, (2007, )1-15.

[29] Marshall, A. W. and Olkin, I., A new method for adding a parameter to a family of distributions with application to the exponential and Weibull families. Biometrika, 84, (1997), 641-652.

[30] Meeker, W.Q. and Escobar, L.A., Statistical Methods for Reliability Data. John Wiley, New York (1998) 
[31] Mudholkar, G. S. and Srivastava, D. K., Exponentiated Weibull family for analyzing bathtub failure-rate data. IEEE Transactions on Reliability, 42, (1993), 299-302.

[32] Nadarajah, S., Kotz, S., Moments of some J-shaped distributions. Journal of Applied Statistics, 30, (2003), 311-317.

[33] Roberts, H. V., Data analysis for managers with minitab. Scientific Press, Redwood City, (1988).

[34] Sharafi M. and Behboodian J., The Balakrishnan skew-normal density. Statistical Papers 49, (2008), 769-778

[35] Zghoul, A. A., Record values from a family of J-shaped distributions. Statistica, 71, (2011), 355-365.

[36] Zhou, M., Yang, D. W., Wang, Y., Nadarajah, S., Some J-shaped distributions: Sums, products and ratios. In Proceedings of the Annual Reliability and Maintainability Symposium, pp. 175-181, (2006).

Current address: Mustafa Korkmaz: Department of Measurement and Evaluation, Artvin ruh University, Artvin, Turkey.

E-mail address: mcagatay@artvin.edu.tr; mustafacagataykorkmaz@gmail.com

ORCID Address: http://orcid.org/0000-0003-3302-0705

Current address: Haitham M. Yousof: Department of Statistics, Mathematics and Insurance, Benha University, Benha, Egypt.

E-mail address: haitham.yousof@fcom.bu.edu.eg

ORCID Address: http://orcid.org/0000-0003-4589-4944

Current address: Morad Alizadeh: Department of Statistics, Persian Gulf University, Bushehr, Iran.

E-mail address: moradalizadeh78@gmail.com

ORCID Address: http://orcid.org/0000-0001-6638-2185

Current address: G.G. Hamedani: Department of Mathematics, Statistics and Computer Science, Marquette University, USA.

E-mail address: gholamhoss.hamedani@marquette.edu

ORCID Address: http://orcid.org/0000-0001-7976-1088 